\title{
The Effect of Vitamin D Supplementation on Blood Lipids in Mi- norities with Type 2 Diabetes
}

Joel C. Exebio ${ }^{1,2}$, Sahar Ajabshir ${ }^{1,3}$, Adriana Campa $^{1}$, Tan Lí ${ }^{4}$, Gustavo G. Zarini' and Fatma G. Huffman ${ }^{1 *}$

${ }^{1}$ Dietetics and Nutrition Department, Robert Stempel College of Public Health and Social Work, Florida International University, USA

${ }^{2}$ Dietetics and Nutrition Department, School of Allied Health, Keiser University, USA

${ }^{3}$ Cellular Biology and Pharmacology Department, Herbert Wertheim College of Medicine, Florida International University, USA

${ }^{4}$ Biostatistics Department, Robert Stempel College of Public Health and Social Work, Florida International University, USA

*Corresponding author: Fatma Huffman, PhD, Dietetics and Nutrition Department, Robert Stempel College of Public Health and Social Work, Florida International University, 11200 SW 8th Street, AHC 5 - 306, Miami, FL 33199, USA, Tel: 1-305-348-3788, Fax: 1-305-348-1996

\begin{abstract}
Introduction: Vitamin D deficiency and type 2 diabetes are common among Hispanics and African Americans in the US. The aim of the study was to determine the effect of supplemental vitamin D intake (4000 IU/day or $6000 \mathrm{IU} /$ day of vitamin D3 over a 6-month period) on blood lipids in a sample of African Americans and Hispanics with type 2 diabetes and vitamin $\mathrm{D}$ insufficiency.

Materials and methods: Participants $(n=75)$ were recruited by community outreach. Participants in both groups were required to take either $4000 \mathrm{IU}$ or $6000 \mathrm{IU}$ of vitamin D (Cholecalciferol) per day given in the form of a pill in a single daily dose. Mixed model was used to compare treatment effects (4000 IU vs. $6000 \mathrm{IU}$ ) on the outcome variables. Bonferroni multiple comparison test was used to detect significant changes from baseline, 3 months, and 6 months.

Results: A significant decrease in total cholesterol (from $193.88 \pm 41.03$ to $180.48 \pm 27.53 \mathrm{mg} / \mathrm{dl}, P=0.040$ ) and triglycerides (from $201.44 \pm 91.35$ to $172.92 \pm 76.87 \mathrm{mg} / \mathrm{dl}, P$ $=0.037$ ) was found for the $6000 \mathrm{IU}$ group at 6 months. The significance was lost after adjusting for confounders.

Conclusion: Our results suggest that the positive effect of vitamin $\mathrm{D}$ supplementation on lipid profile may be mediated by other cofactors related to vitamin $\mathrm{D}$ metabolism among $\mathrm{Hi}-$ spanic and African American participants with type 2 diabetes.
\end{abstract}

\section{Keywords}

Vitamin D, Blood lipids, Hispanics, African Americans, Type 2 diabetes

\section{Introduction}

Low levels of vitamin D are prevalent in the US. ACcording to data from the National Health and Nutrition Examination Surveys (NHANES) in 2001-2006, 33\% of the general US population had serum 25(OH)D below $50 \mathrm{nmol} / \mathrm{L}$. In addition, low vitamin D levels are common among minorities. For instance, $41 \%$ of African Americans and $33 \%$ of Mexican Americans had serum 25(OH)D below $50 \mathrm{nmol} / \mathrm{L}$ compared to $18 \%$ of non- $\mathrm{Hi}-$ spanic Whites [1].

Vitamin $D$ is produced in the skin by the action of ultraviolet light. Even with similar levels of sun exposure, the skin color is a key determinant in vitamin $\mathrm{D}$ production. African Americans and Hispanics are at a particularly high risk for vitamin $D$ deficiency because their darker skin color limits the amount of ultraviolet light that penetrates, thereby reducing cutaneous synthesis of vitamin $D_{3}$ [2]. Vitamin $D$ can also be obtained from the diet with consumption of vegetable and animal products. Vitamin $D$ needs to be activated by hydroxylation in the liver and kidney to form its metabolically active form: $1,25(\mathrm{OH})_{2} \mathrm{D}_{3}$. This metabolite produces its effects by activating the vitamin D receptor (VDR) in the cells [2].

The VDR is present in vascular and myocardial cells

Citation: Exebio JC, Ajabshir S, Campa A, Li T, Zarini GG, et al. (2018) The Effect of Vitamin D Supplementation on Blood Lipids in Minorities with Type 2 Diabetes. Int J Diabetes Clin Res 5:093. doi. org/10.23937/2377-3634/1410093

Accepted: September 27, 2018: Published: September 29, 2018

Copyright: (c) 2018 Exebio J, et al. This is an open-access article distributed under the terms of the Creative Commons Attribution License, which permits unrestricted use, distribution, and reproduction in any medium, provided the original author and source are credited. 
which suggest an involvement of vitamin $D$ mediated effects in the development of cardiovascular disease (CVD) [3]. Cardiovascular risk factors such as type 2 diabetes (T2D) and dyslipidemia are the major causes of death in the US, especially among minorities [4]. Therefore, it is of crucial importance to determine the role of vitamin D supplementation on the prevention of CVD.

Associations among vitamin D insufficiency and lower high density lipoprotein (HDL), higher triglycerides, and hypercholesterolemia have been reported [57]. However, randomized clinical trials evaluating the effects of vitamin $D$ repletion on lipid panel have reported conflicting results [8-10].

Patients with T2D are more likely to develop hypertension, dyslipidemia, arteriosclerosis and all of the complications related to myocardial infarction and stroke [11]. Since finding new strategies to decrease diabetes complications and cardiovascular risk among patients with T2D is a major public health goal, testing the direct benefit of vitamin $D$ repletion on blood lipids is warranted. In addition, most of the current studies have been conducted in Caucasian populations. Since African Americans and Hispanics are more prone to both vitamin $D$ insufficiency and T2D compared to Caucasians, it is important to test the effect of vitamin $D$ repletion on these high risk populations.

The aim of the present study was to determine the effect of supplemental vitamin D intake (4000 IU/day or $6000 \mathrm{IU} /$ day of vitamin $D_{3}$ over a 6 -month period) on blood lipids in a sample of African Americans and Hispanics with T2D and vitamin D insufficiency $(25(\mathrm{OH}) \mathrm{D}<30$ $\mathrm{ng} / \mathrm{ml}$ ) living in South Florida.

\section{Methods}

\section{Subject recruitment}

An intervention study to assess the effects of vitamin D supplementation (4000 IU/day and $6000 \mathrm{IU} /$ day) over six months on cardiovascular disease markers among a sample of African Americans and Hispanics with T2D was conducted (clinicaltrials.gov NCT 01412710). The present study analyzed the data collected at screening (serum 25(OH)D), baseline (blood lipids), 3 months (serum 25(OH)D and blood lipids), and 6 months (serum 25(OH)D and blood lipids).

The study consisted of two phases: phase one included the screening of individuals, phase two included the recruitment of individuals who met inclusion criteria and beginning of the vitamin $D$ intervention trial. Inclusion criteria were vitamin $D$ insufficiency $(25(\mathrm{OH}) \mathrm{D}<30$ $\mathrm{ng} / \mathrm{ml}), 30-70$ years of age, African American or Hispanic with confirmed T2D. Exclusion criteria were taking vitamin D supplements other than standard daily multivitamin formula, receiving insulin therapy, being pregnant or lactating, having thyroid, hepatic, renal dysfunction, cancer, HIV, or major psychiatric disorders. Renal fail- ure was defined as currently on dialysis or glomerular filtration rate lower than $15 \mathrm{ml} / \mathrm{min} / 1.73 \mathrm{~m}^{2}$ (stage 5) confirmed by primary care physician.

Flyers explaining the purpose of the study, inclusion/exclusion criteria and the investigators' emails and phone numbers were distributed in highly visited community areas such as churches, supermarkets, and clinics. Two clinics were visited every week for recruitment purposes: Borinquen Health Care Center and Clinical Care Medical Center. When volunteer participants called the investigators back, purposes of the study were explained and specific questions were asked to assure compliance with inclusion criteria. All qualified participants were invited to take part in a morning session at the Human Nutrition Laboratory at Florida International University (FIU) for fasting blood sampling to screen for vitamin $D$ insufficiency. The study was approved by IRB at FIU. All participants were asked to sign an informed consent form previous to screening data collection. Only participants with confirmed T2D diagnosis by a physician were eligible for the study. Exclusion criteria were self-reported and confirmed by primary care physician. All participants were screened for vitamin $D$ insufficiency in phase one. They were contacted either to continue in the study or to inform them that they were disqualified based on their vitamin $\mathrm{D}$ levels.

Participants in both groups were required to take either $4000 \mathrm{IU}$ or $6000 \mathrm{IU}$ of vitamin D (Cholecalciferol) per day given in the form of a pill in a single daily dose. For safety reasons, the group receiving the higher dosage was proportionally half the size of the group receiving 4000 IU per day. Therefore, less patients were exposed to possible toxic effects. Subjects were included in a first come, first served basis and assigned in a 2:1 proportion to the $4000 \mathrm{IU}$ or $6000 \mathrm{IU}$ group until minimum sample size needed was achieved. For example, the first and second subjects that qualified for the study were assigned to the $4000 \mathrm{IU}$ group, the third subject to the 6000 IU group. The study was not blinded. Compliance was determined by returned pill count at follow ups. When less than $80 \%$ of the pills were taken, the subject was classified as non-compliant. Adverse events were recorded at each visit. Each participant was seen 4 times; at screening, baseline, 3 months, and 6 months. Serum 25(OH)D was measured at screening, 3 months, and 6 months. Blood lipids were measured at baseline, 3 months, and 6 months. The time spam between screening and baseline was one week.

Total study lasted for 25 weeks for each participant from screening to final assessment. The study was conducted across all seasons. The study started in June 2011 and was completed on September 2013. In addition, located in a tropical area, sunlight is plentiful in South Florida all year round. Therefore, all subjects had similar sun exposure potential. Ninety-two participants were screened. Seventy-five participants met the study`s in- 
clusion ( $\mathrm{n}=50$ for $4000 \mathrm{IU} /$ day treatment and $\mathrm{n}=25$ for $6000 \mathrm{IU} /$ day treatment). Three participants qualified for the study based on their vitamin $D$ status but did not attend the 3 month and 6 month follow ups (all in the 4000 IU group). Eight participants attended the 3 month but not the 6 month follow up ( 5 in the $4000 \mathrm{IU}$ and 3 in the 6000 IU group, respectively). Five participants completed the study but were non-compliant ( 3 in the 4000 IU and 2 in the 6000 IU treatment, respectively). Since an intent to treat approach was followed, all 75 participants were included in the present study.

\section{Blood collection}

Venous blood (15 ml) was collected from each subject by a certified phlebotomist using standard laboratory methods at each study point. After coagulation, blood was centrifuged at 2500 RPM for 30 minutes. Plasma total cholesterol, triglycerides, LDL, and HDL cholesterol were assayed by enzymatic methods (Cobas Mira, Roche Diagnostics, Indianapolis, IN). Serum vitamin D concentrations were measured with an enzymeimmunoassay kit by absorbance (Immunodiagnostic Systems Scottsdale, AZ). Vitamin D values were tested at screening, 3-month and 6-month visits. Total cholesterol, triglycerides, LDL, and HDL cholesterol were tested at baseline, 3-month, and 6-month visits.

\section{Socio-demographic questionnaire}

Participants were asked to complete the standard questionnaire on site. Trained bilingual interviewers (English and Spanish) administered the questionnaires in the subjects' language of preference. Data were collected using a socio-demographic questionnaire which included questions related to gender, age, education level, years with diabetes, smoking, and medication use.

\section{Anthropometric measurements}

Height and weight were measured using a SECA clinical balance scale (Seca Corp, Columbia, MD). Body mass index (BMI) was calculated as weight in $\mathrm{kg} / \mathrm{height}$ in $\mathrm{m}^{2}$. BMI was calculated at baseline, 3-month, and at 6-month visits. Only baseline values were used for the analysis due to the lack of significant changes in BMI at 3 months and 6 months.

\section{Sun exposure questionnaire}

The sun exposure questionnaire developed by Hanwell, et al. [12] was applied following the original rubric. Time spent outdoors during the previous week $0 \leq 5 \mathrm{~min}$ utes, $1=5-30$ minutes and $2 \geq 30 \mathrm{~min}$ ) was self-reported. Four options for skin exposed while outdoors were offered ( 1 = face and hands, 2 = face, hands and arms, 3 = face, hands and legs and $4=$ bathing suit). The daily sun exposure score for each day was calculated by multiplying the time spent outdoors score times the skin exposed while outdoors score. The scale for each day ranged from 0 to 8 . The weekly sun exposure was calculated by adding the daily scores ( $\min =0, \max =56$ ). The questionnaire was administered at baseline, 3 months, and 6 months, but because sun exposure scores did not change significantly, only baseline values were used in the analysis.

\section{Skin color}

In order to have an objective measure of sun exposure, skin color was determined by reflectance colorimetry using the SmartProbe 400 (IMS Inc. USA). This instrument uses the International Commission on Illumination Scale which ranges from 0 (black) to 100 (white) for skin color. Two readings at each measurement site for each participant were taken: two on the dorsal aspect of the wrist of the right hand (area most exposed to sun), two on the inside of the right upper arm (area less exposed to the sun). The mean values of the two readings at each measurement site were used for analysis. Data were collected at baseline, 3 months, and 6 months, but because it did not change significantly, only baseline values were used in the present analysis. Since participants with darker skin color tend to have lower levels of serum $25(\mathrm{OH}) \mathrm{D}$, skin color may have an effect on lipid profile.

\section{Assessment of dietary intake}

Dietary intake was determined using the long food frequency questionnaire (FFQ) developed by Walter Willett [13]. This FFQ has been validated in different ethnic groups and, also, specifically in Cuban-Americans in South Florida [14]. Participants self-reported the average consumption of specific amount of foods over the past 12 months. Frequencies ranged from "never" to "six or more servings per day". The FFQ also assessed the frequency use of multivitamin/mineral supplements, salt, sugar, alcohol and vitamin $D$ intake. The questionnaire was administered at baseline.

\section{Statistical analysis}

Descriptive statistics were used for baseline characteristics. Numerical normally distributed data and categorical data were compared using independent t-test and Chi-Squared test, respectively. Intent to treat analysis was used to analyze the effect of vitamin D on the outcome variables.

Main variables of the study were: plasma total cholesterol, triglycerides, LDL, and HDL cholesterol. All variables were input as continuous in the analysis. Control variables included: BMI, gender, age, smoking, years with diabetes, sun exposure score, vitamin $D$ intake, fasting plasma glucose, lipid lowering medication use, and skin color.

Mixed model was used to compare treatment effects ( $4000 \mathrm{IU}$ vs. $6000 \mathrm{IU}$ ) on the outcome variables. Bonferroni multiple comparison test was used to detect significant changes from baseline, 3 months, and 6 months on the outcome variables with and without adjustment for age, gender, years with diabetes, smoking, BMI, vitamin $D$ intake, sun exposure score, upper arm skin color, forearm skin color, fasting plasma glucose, and li- 
Table 1: Baseline characteristics of participants prior to vitamin D repletion treatments.

\begin{tabular}{|c|c|c|c|}
\hline \multirow[t]{2}{*}{ Characteristic } & \multicolumn{2}{|l|}{ Treatment } & \multirow[t]{2}{*}{$P$-value } \\
\hline & $4000 \mathrm{IU}(\mathrm{n}=50)$ & $6000 \mathrm{IU}(\mathrm{n}=25)$ & \\
\hline 25(OH)D (ng/ml) & $22.25 \pm 7.19$ & $23.74 \pm 5.09$ & 0.302 \\
\hline FGP (mg/dl) & $188.76 \pm 87.19$ & $168.16 \pm 55.94$ & 0.220 \\
\hline Age (years) & $54.76 \pm 8.56$ & $54.17 \pm 10.99$ & 0.999 \\
\hline Gender (Female \%) & $48 \%$ & $52 \%$ & 0.809 \\
\hline Years with T2D & $6.48 \pm 5.18$ & $5.48 \pm 5.93$ & 0.478 \\
\hline Smoking (yes \%) & $10 \%$ & $28 \%$ & 0.091 \\
\hline BMI $\left(\mathrm{kg} / \mathrm{m}^{2}\right)$ & $34.72 \pm 6.98$ & $30.74 \pm 4.93$ & 0.013 \\
\hline Total Cholesterol (mg/dl) & $213.08 \pm 42.30$ & $193.88 \pm 41.03$ & 0.065 \\
\hline Triglycerides (mg/dl) & $182.98 \pm 110.11$ & $201.44 \pm 91.35$ & 0.445 \\
\hline LDL cholesterol (mg/dl) & $128.34 \pm 40.54$ & $115.56 \pm 34.53$ & 0.160 \\
\hline HDL cholesterol (mg/dl) & $50.64 \pm 12.20$ & $42.44 \pm 10.40$ & 0.004 \\
\hline Vitamin D intake (IU/day) & $316.82 \pm 251.70$ & $301.97 \pm 225.49$ & 0.797 \\
\hline Sun exposure score & $20.06 \pm 14.92$ & $18.68 \pm 15.52$ & 0.715 \\
\hline Upper arm skin color & $58.67 \pm 9.54$ & $62.09 \pm 5.65$ & 0.056 \\
\hline Forearm skin color & $54.82 \pm 8.51$ & $54.37 \pm 4.37$ & 0.099 \\
\hline Lipid lowering meds & $64 \%$ & $68 \%$ & 0.215 \\
\hline
\end{tabular}

Continues variables are presented as mean \pm SD and categorical variables as $\%$.

25(OH)D: serum vitamin D; FPG: fasting plasma glucose; T2D: type 2 diabetes; BMI: body mass index; LDL: low density lipoprotein; HDL: high density lipoprotein.

Table 2: Group comparisons of metabolic parameters at different time points without adjusting for covariates.

\begin{tabular}{|c|c|c|}
\hline & $4000 \mathrm{IU}(n=50)$ & $6000 \mathrm{IU}(\mathrm{n}=25)$ \\
\hline \multicolumn{3}{|l|}{$25(\mathrm{OH}) \mathrm{D}(\mathrm{ng} / \mathrm{ml})$} \\
\hline Baseline & $22.25 \pm 7.19$ & $23.74 \pm 5.09$ \\
\hline 3 months & $37.34 \pm 12.31^{*}$ & $43.20 \pm 15.94^{*}$ \\
\hline 6 months & $37.99 \pm 13.22^{*}$ & $40.62 \pm 19.39^{*}$ \\
\hline$P$-value groups $=0.225$ & $P$-value time $=0.006$ & $P$-value interaction $=0.238$ \\
\hline \multicolumn{3}{|l|}{ Total Cholesterol (mg/dl) } \\
\hline Baseline & $213.08 \pm 42.30$ & $193.88 \pm 41.03$ \\
\hline 3 months & $209.26 \pm 43.62$ & $184.68 \pm 50.89$ \\
\hline 6 months & $199.84 \pm 32.79$ & $180.48 \pm 27.53^{*}$ \\
\hline$P$-value groups $=0.027$ & $P$-value time $=0.044$ & $P$-value interaction $=0.653$ \\
\hline \multicolumn{3}{|l|}{ Triglycerides (mg/dl) } \\
\hline Baseline & $182.98 \pm 110.11$ & $201.44 \pm 91.35$ \\
\hline 3 months & $174.62 \pm 109.83$ & $192.20 \pm 94.49$ \\
\hline 6 months & $187.08 \pm 151.26$ & $172.92 \pm 76.87^{\star}$ \\
\hline$P$-value groups $=0.221$ & $P$-value time $=0.117$ & $P$-value interaction $=0.227$ \\
\hline \multicolumn{3}{|l|}{ LDL Cholesterol } \\
\hline Baseline & $128.34 \pm 40.54$ & $115.56 \pm 34.53$ \\
\hline 3 months & $125.88 \pm 40.90$ & $120.00 \pm 35.37$ \\
\hline 6 months & $120.76 \pm 30.35$ & $121.96 \pm 40.60$ \\
\hline$P$-value groups $=0.542$ & $P$-value time $=0.685$ & $P$-value interaction $=0.354$ \\
\hline \multicolumn{3}{|l|}{ HDL Cholesterol } \\
\hline Baseline & $50.64 \pm 12.20$ & $42.44 \pm 10.40$ \\
\hline 3 months & $48.92 \pm 11.12$ & $42.12 \pm 12.35$ \\
\hline 6 months & $46.98 \pm 10.37$ & $42.12 \pm 12.76$ \\
\hline$P$-value groups $=0.072$ & $P$-value time $=0.214$ & $P$-value interaction $=0.351$ \\
\hline
\end{tabular}

Data represented by mean \pm standard error; "Represents significant differences from baseline; Level of significance is at $P<0.05$. 25(OH)D: serum vitamin D; LDL: low density lipoprotein; HDL: high density lipoprotein.

pid lowering medication use. Significance was set at $p<$ 0.05 and all analyses were two sided. Statistical analysis was conducted using SPSS 18.0 (Chicago).

\section{Results}

Groups were different at baseline on BMI (34.72 \pm
$6.98 \mathrm{~kg} / \mathrm{m}^{2}$ vs. $30.74 \pm 4.93 \mathrm{~kg} / \mathrm{m}^{2}, P=0.013$ for $4000 \mathrm{IU}$ and $6000 \mathrm{IU}$; respectively) and HDL cholesterol (50.64 \pm $12.20 \mathrm{mg} / \mathrm{dl}$ vs. $42.44 \pm 10.40 \mathrm{mg} / \mathrm{dl}, P=0.004$ for 4000 IU and $6000 \mathrm{IU}$; respectively) (Table 1).

There was no significant difference between groups 
regarding serum 25(OH)D ( $P=0.225)$. However, significant improvements in serum $25(\mathrm{OH}) \mathrm{D}$ levels were seen from baseline to 3 month and 6 months, respectively in both treatments (from $22.25 \pm 7.19$ to $37.34 \pm 12.31$ and $37.99 \pm 13.22, P<0.05$ in the 4000 IU group; from 23.74 \pm 5.09 to $43.20 \pm 15.94$ and $40.62 \pm 19.39, P<0.05$ in the 6000 IU group) (Table 2).

Differences between 3 months and 6 months' levels for serum 25(OH)D were not significant in both treatments $(P>0.05)$. In the 6000 IU group, the value showed a non-significant decline at 6 months compared to the 3 months follow up.

In the unadjusted model, the difference between and within groups was significant for total cholesterol $(P=0.027$ and $P=0.044$, respectively). However, the interaction between time and treatment was not significant $(P=0.653)$ (Table 2). The multiple comparisons analysis showed a borderline not significant decrease in total cholesterol for the $4000 \mathrm{IU}$ group at 6 months (from $213.08 \pm 42.30$ to $199.84 \pm 32.79 \mathrm{mg} / \mathrm{dl}, P=0.052$ ) and a significant decrease for the $6000 \mathrm{IU}$ group at 6 months (from $193.88 \pm 41.03$ to $180.48 \pm 27.53 \mathrm{mg} / \mathrm{dl}$, $P=0.040)$. However, when the model was adjusted for confounders, significance was lost (Table 3).

Similarly, in the unadjusted model (Table 2), a significant change for triglycerides was observed at 6 months in the $6000 \mathrm{IU}$ group (from $201.44 \pm 91.35$ to $172.92 \pm$ $76.87 \mathrm{mg} / \mathrm{dl}, P=0.037)$. The significance was lost after adjusting for confounders (Table 3 ).

In the adjusted model, only serum 25(OH)D changed significantly over time (Table 3). Adherence defined as consumption of more than $80 \%$ of supplied vitamin D pills was $94 \%$ for the 4000 IU group and $92 \%$ for the $6000 \mathrm{IU}$ group without any differences between groups. No adverse events were reported during the course of the study.

Three participants stopped taking lipid lowering medication during the course of the study due to lack of money to buy the refills ( 2 in the 4000 IU group and 1 in the 6000 IU group). No other changes in medication dosage were reported.

\section{Discussion}

In the current study, vitamin $D_{3}$ supplementation at $6000 \mathrm{IU} /$ day significantly reduced total cholesterol, and triglycerides in a sample of Hispanics and African Americans with T2D and vitamin D insufficiency. However, the significance was lost after adjusting for confounders.

In fact, vitamin D insufficiency is related to other cardiovascular risk factors such as obesity, smoking, and unhealthy diet $[15,16]$. Regarding the strong association between obesity and vitamin D insufficiency, it has been

Table 3: Group comparisons of metabolic parameters at different time points adjusting for covariates.

\begin{tabular}{|c|c|c|}
\hline & $4000 \mathrm{IU}(n=50)$ & $6000 \mathrm{IU}(n=25)$ \\
\hline \multicolumn{3}{|l|}{$25(\mathrm{OH}) \mathrm{D}(\mathrm{ng} / \mathrm{ml})$} \\
\hline Baseline & $22.25 \pm 7.19$ & $23.74 \pm 5.09$ \\
\hline 3 months & $37.34 \pm 12.31^{\star}$ & $43.20 \pm 15.94^{*}$ \\
\hline 6 months & $37.99 \pm 13.22^{*}$ & $40.62 \pm 19.39^{*}$ \\
\hline$P$-value groups $=0.232$ & $P$-value time $=0.019$ & $P$-value interaction $=0.352$ \\
\hline \multicolumn{3}{|l|}{ Total Cholesterol (mg/dl) } \\
\hline Baseline & $213.08 \pm 42.30$ & $193.88 \pm 41.03$ \\
\hline 3 months & $209.26 \pm 43.62$ & $184.68 \pm 50.89$ \\
\hline 6 months & $199.84 \pm 32.79$ & $180.48 \pm 27.53$ \\
\hline$P$-value groups $=0.204$ & $P$-value time $=0.954$ & $P$-value interaction $=0.605$ \\
\hline \multicolumn{3}{|l|}{ Triglycerides (mg/dl) } \\
\hline Baseline & $182.98 \pm 110.11$ & $201.44 \pm 91.35$ \\
\hline 3 months & $174.62 \pm 109.83$ & $192.20 \pm 94.49$ \\
\hline 6 months & $187.08 \pm 151.26$ & $172.92 \pm 76.87$ \\
\hline$P$-value groups $=0.962$ & $P$-value time $=0.250$ & $P$-value interaction $=0.167$ \\
\hline \multicolumn{3}{|l|}{ LDL Cholesterol } \\
\hline Baseline & $128.34 \pm 40.54$ & $115.56 \pm 34.53$ \\
\hline 3 months & $125.88 \pm 40.90$ & $120.00 \pm 35.37$ \\
\hline 6 months & $120.76 \pm 30.35$ & $121.96 \pm 40.60$ \\
\hline$P$-value groups $=0.969$ & $P$-value time $=0.872$ & $P$-value interaction $=0.423$ \\
\hline \multicolumn{3}{|l|}{ HDL Cholesterol } \\
\hline Baseline & $50.64 \pm 12.20$ & $42.44 \pm 10.40$ \\
\hline 3 months & $48.92 \pm 11.12$ & $42.12 \pm 12.35$ \\
\hline 6 months & $46.98 \pm 10.37$ & $42.12 \pm 12.76$ \\
\hline$P$-value groups $=0.088$ & $P$-value time $=0.188$ & $P$-value interaction $=0.118$ \\
\hline
\end{tabular}

Data represented by mean \pm standard error. "Represents significant differences from baseline. Level of significance is at $P<0.05$. 25(OH)D: serum vitamin D; LDL: low density lipoprotein; HDL: high density lipoprotein; Analysis was adjusted for age, gender, years with diabetes, smoking, BMI, vitamin $\mathrm{D}$ intake, sun exposure score, fasting plasma glucose, upper arm skin color, forearm skin color, and lipid lowering medication use. 
hypothesized that vitamin D is stored in the fat tissue and is not bioavailable in obese participants resulting in low levels of serum vitamin D despite sufficient intake [15]. In this context, if obesity is corrected we may see improvements in both serum vitamin D levels and lipid profile. Our results support this hypothesis. The participants were obese based on BMI classification. The positive effect of vitamin $D$ supplementation on total cholesterol and triglycerides observed in the $6000 \mathrm{IU} /$ day group disappeared when the model was adjusted for covariates such as smoking, age, gender, skin color, sun exposure, lipid lowering medication, years with diabetes, and BMI. Therefore, our results suggest that the association between vitamin $D$ insufficiency and dyslipidemia may be mediated by other cardiovascular risk factors.

Recent clinical trials examining the effect of vitamin D supplementation on lipid profile among individuals with and without T2D and vitamin D insufficiency have been inconsistent $[8,9,17]$. Some studies have found significant improvements in lipid profile after vitamin D supplementation. However, none of them adjusted for covariates in the analysis. Alkharfy, et al. [9], randomly assigned 499 participants with T2D to 8 groups: control (n $=151)$, rosiglitazone alone $(n=49)$, diet $(n=15)$, insulin alone $(n=55)$, insulin + orals $(n=12)$, metformin alone $(n=121)$, oral agents combination $(n=37)$, and sulphonylurea alone $(n=59)$. Participants received $2000 \mathrm{IU} /$ day of vitamin $D_{3}$ for 12 months. In the insulin alone group, total cholesterol decreased significantly in women. In the insulin + orals group, triglycerides and total cholesterol decreased significantly after 12 months. This study provides a view of how vitamin $D$ supplementation can interact with certain drugs commonly used in patients with T2D regarding lipid lowering benefits. In contrast, Ponda, et al. [8], randomly assigned 151 vitamin D deficient participants at high risk for CVD to either 50,000 IU of vitamin $D_{3}$ weekly or placebo. Vitamin $D$ failed to improve lipid profile. In addition, a significant increase in LDL was noted which significantly correlated with a decrease in parathyroid hormone (PTH) levels. Similarly, Salehpour, et al. [17], randomly distributed 77 healthy premenopausal overweight and obese women to either $25 \mu \mathrm{g} /$ day of vitamin $D_{3}$ or placebo for 12 weeks. Significant increases in total cholesterol, LDL and HDL were reported. This study excluded participants taking any antihypertensive and lipid lowering medication. Since it is difficult to evaluate the effect of vitamin D supplementation on lipid profile when participants are already taking lipid lowering medication, this study provided a new insight into the raw metabolic effects of vitamin $D$. Authors from the last two studies warned that vitamin D may have both positive and negative effects on different cholesterol particles. Therefore, it should be monitored and used with caution among participants at risk of CVD. Conflicting results may be a consequence of different dosages used, different populations, different duration of treatment, and lack of control for obesity, physical activity, sun exposure, vitamin D intake, and medication usage.

In a meta-analysis of 10 randomized controlled trials conducted by Wang, et al. [18], obesity was a strong confounding variable. When subjects were classified according to $\mathrm{BMI}>30 \mathrm{~kg} / \mathrm{m}^{2}$, supplementation with vitamin D showed a negative effect on lipid profile compared to non-obese subjects. For instance, total cholesterol increased $3.22 \mathrm{mg} / \mathrm{dl}$ in the obese group compared with a reduction of $0.68 \mathrm{mg} / \mathrm{dl}$ in the non-obese group. Even though, these results were not statistically significant, a trend was observed. Authors concluded that none of the 10 studies reviewed in the meta-analysis were specifically designed to detect changes in lipid profile and, therefore, were lacking power. Also, all the studies used low dosages of vitamin D (from $300 \mathrm{IU}$ to $3332 \mathrm{IU}$ daily), and hyperlipemia and vitamin $D$ deficiency were not inclusion criteria which decreases the probabilities of finding significant changes at the end of the treatments. Similarly, none of the 10 studies included were conducted on Hispanics or African Americans.

Another meta-analysis of 12 randomized controlled trials conducted by Swart, et al. [19], found significant changes in LDL only in participants with the lowest baseline serum vitamin D. Total cholesterol also decreased but the change was not statistically significant due to lack of power. However, the authors remarked that the change was clinically significant. Authors concluded that none of the studies recruited participants based on their vitamin $D$ status, resulting in low number of subjects with vitamin $D$ deficiency, leading to negative effects of vitamin D supplementation on lipid profile. Also, all the studies were conducted among healthy Caucasian populations.

Regarding lipid metabolism, studies have shown that vitamin D can reduce hepatic triglyceride synthesis and increase uptake by peripheral tissues due to increased intestinal calcium absorption $[20,21]$. Similarly, vitamin D promotes the formation of large HDL particles due to an increase in apolipoprotein $A 1$, which increases reverse cholesterol transport [22]. Vitamin D also increases the lipolytic activity of heparin and reduces VLDLcholesterol synthesis [20].

In our study, the positive effect of vitamin D supplementation on total cholesterol and triglycerides was observed only in the 6000 IU group despite both groups reaching similar serum vitamin $D$ levels. It may be that sufficient vitamin D status was achieved sooner in the 6000 IU/group. Therefore, participants were exposed to sufficient vitamin $D$ for a longer period of time. The halflife of vitamin $D_{3}$ is about 2 months. Therefore, maintaining a plateau of sufficient $25(\mathrm{OH}) \mathrm{D}$ levels for more than 20 weeks is needed in order to see improvements on tissues not related to calcium metabolism [23].

Our study has several strengths. First, to our knowl- 
edge this is the first study that analyzed vitamin D supplementation effects on Hispanics and African Americans with T2D living in a tropical area like South Florida. Second, the dosages used were large enough to raise serum vitamin $D$ to normal levels in a population that was insufficient at baseline. Third, analysis was adjusted for a wide range of covariates related to vitamin D metabolism. Fourth, only participants with vitamin D insufficiency and confirmed T2D were included. Lastly, compliance in our study was high with $94 \%$ for the 4000 IU group and $92 \%$ for the 6000 IU group without any differences between groups. No adverse events were reported during the course of the study.

Limitations of our study included. First, participants were not randomly distributed among groups as evidenced by differences in BMI and HDL cholesterol at baseline. Second, a longer duration of treatment may be needed to see more drastic effects on lipid profile. In addition, the number of African American participants was not large enough to analyze data by ethnicity. Lastly, $64 \%$ and $68 \%$ of participants were taking lipid lowering medication in the $4000 \mathrm{IU}$ and $6000 \mathrm{IU}$ groups, respectively. Medication use may mask the effect of vitamin D supplementation. We tried to control for it by advising participants to keep their medication use constant during the study. However, this panorama reflects common clinical practice among patients with T2D.

Finally, our results suggest that the positive effect of vitamin $D$ supplementation on lipid profile may be mediated by other cofactors related to vitamin D metabolism among Hispanic and African American participants with T2D. Longer, well designed randomized clinical trials are needed to confirm these results.

\section{Acknowledgments}

This research was funded by a grant from NIH/MBRS/ SCORE AC\# 124401529/42. Joel Exebio was supported by NIH/NIGMS R25 GM061347.

\section{Disclosure}

No potential conflict of interest relevant to this article was reported.

\section{References}

1. Anne C Looker, Clifford L Johnson, David A Lacher, Christine M Pfeiffer, Rosemary L Schleicher, et al. (2013) Vitamin D Status: United States, 2001-2006. Centers for Disease Control and Prevention.

2. Holick MF (2003) Vitamin D: A millennium perspective. J Cell Biochem 88: 296-307.

3. Muller DN, Kleinewietfeld M, Kvakan H (2011) Vitamin D review. J Renin Angiotensin Aldosterone Syst 12: 125-128.

4. World Health Organization (2012) World Health Statistics 2012. Geneva, Switzerland.

5. Jorde R, Grimnes G (2011) Vitamin D and metabolic health with especial reference to the effect of vitamin $D$ on serum lipids. Prog Lipid Res 50: 303-312.

6. Jaimungal S, Wehmeier K, Mooradian AD, Haas MJ (2011)
The emerging evidence for vitamin D-mediated regulation of apolipoprotein A1 synthesis. Nutr Res 31: 805-812.

7. Skaaby T, Husemoen LLN, Pisinger $C$, Jorgensen $T$, Thuesen $\mathrm{BH}$, et al. (2012) Vitamin D status and changes in cardiovascular risk factors: A prospective study of a general population. Cardiology 123: 62-70.

8. Ponda MP, Dowd K, Finkielstein D, Holt PR, Breslow JL (2012) The short-term effects of vitamin D repletion on cholesterol: A randomized, placebo-controlled trial. Arterioscler Thromb Vasc Biol 32: 2510-2515.

9. Alkharfy KM, Al-Daghri NM, Sabico SB, Al-Othman A, Moharram O, et al. (2013) Vitamin D supplementation in patients with diabetes mellitus type 2 on different therapeutic regimens: A one-year prospective study. Cardiovasc Diabetol 12: 113-123.

10. Al-Daghri NM, Alkharfy KM, El-Kholie E, Yousef M, Al-Othman A, et al. (2012) Vitamin D supplementation as an adjuvant therapy for Saudi patients with DMT2: An 18-month interventional study. Cardiovasc Diabetol 11: 1-7.

11. Ginsberg HN (2000) Insulin resistance and cardiovascular disease. J Clin Invest 106: 453-458.

12. Hanwell HE, Vieth R, Cole DE, Scillitani A, Modoni S, et al. (2010) Sun exposure questionnaire predicts circulating 25-hydroxyvitamin $D$ concentrations in Caucasian hospital workers in southern Italy. J Steroid Biochem Mol Biol 121: 334-337.

13. Willett WC, Sampson L, Stampfer MJ, Rosner B, Bain C, et al. (1985) Reproducibility and validity of a semiquantitative food frequency questionnaire. Am J Epidemiol 122: 51-65.

14. Nath SD, Huffman FG (2005) Validation of a semi quantitative food frequency questionnaire to assess energy and macronutrient intakes of Cuban Americans. Int J Food Sci Nutr 56: 309-314.

15. Thuesen B, Husemoen L, Fenger M, Jakobsen J, Schwarz $P$, et al. (2012) Determinants of vitamin $D$ status in a general population of Danish adults. Bone 50: 605-610.

16. Borges MC, Martini LA, Rogero MM (2011) Current perspectives on vitamin $\mathrm{D}$, immune system, and chronic diseases. Nutrition 27: 399-404.

17. Salehpour A, Shidfar F, Hosseinpanah F, Vafa M, Razaghi $M$, et al. (2012) Vitamin $D_{3}$ and the risk of CVD in overweight and obese women: $\mathrm{A}$ randomized controlled trial. $\mathrm{Br} \mathrm{J}$ Nutr 108: 1866-1873.

18. Wang H, Xia N, Yang Y, Peng D (2012) Influence of vitamin D supplementation on plasma lipid profiles: A meta-analysis of randomized controlled trials. Lipids Health Dis 11: 42.

19. Swart K, Lips P, Brouwer I, Jorde R, Heymans M, et al. (2018) Effect of vitamin D supplementation on markers of cardiovascular disease and type 2 diabetes: An individual participant data meta-analysis of randomized controlled trials. Am J Clin Nutr 107: 1043-1053.

20. Khajehdehi P, Taheri S (2003) Effect of oral calcitriol pulse therapy on the lipid, calcium, and glucose homeostasis of hemodialysis patients: Its safety in a combination with oral calcium carbonate. J Ren Nutr 13: 78-83.

21. Lacour B, Basile C, Drueke T, Funck-Brentano JL (1982) Parathyroid function and lipid metabolism in the rat. Miner Electrolyte Metab 7: 157-165.

22. Ogata T, Miyauchi T, Sakai S, Irukiyama-Tomobe Y, Goto $\mathrm{K}$, et al. (2002) Stimulation of peroxisome-proliferator-activated receptor alpha (PPAR alpha) attenuates cardiac fibrosis and endothelin-1 production in pressure-overloaded rat hearts. Clin Sci (Lond) 103: S284-S288.

23. Jones $G$ (2008) Pharmacokinetics of vitamin D toxicity. Am J Clin Nutr 88: S582-S586. 